Document downloaded from:

http://hdl.handle.net/10251/78058

This paper must be cited as:

Sanguesa, JA.; Fogue, M.; Garrido, P.; Martínez, FJ.; Cano Escribá, JC.; Tavares De Araujo Cesariny Calafate, CM.; Manzoni, P. (2013). On the Selection of Optimal Broadcast Schemes in VANETs. ACM. doi:10.1145/2507924.2507935.

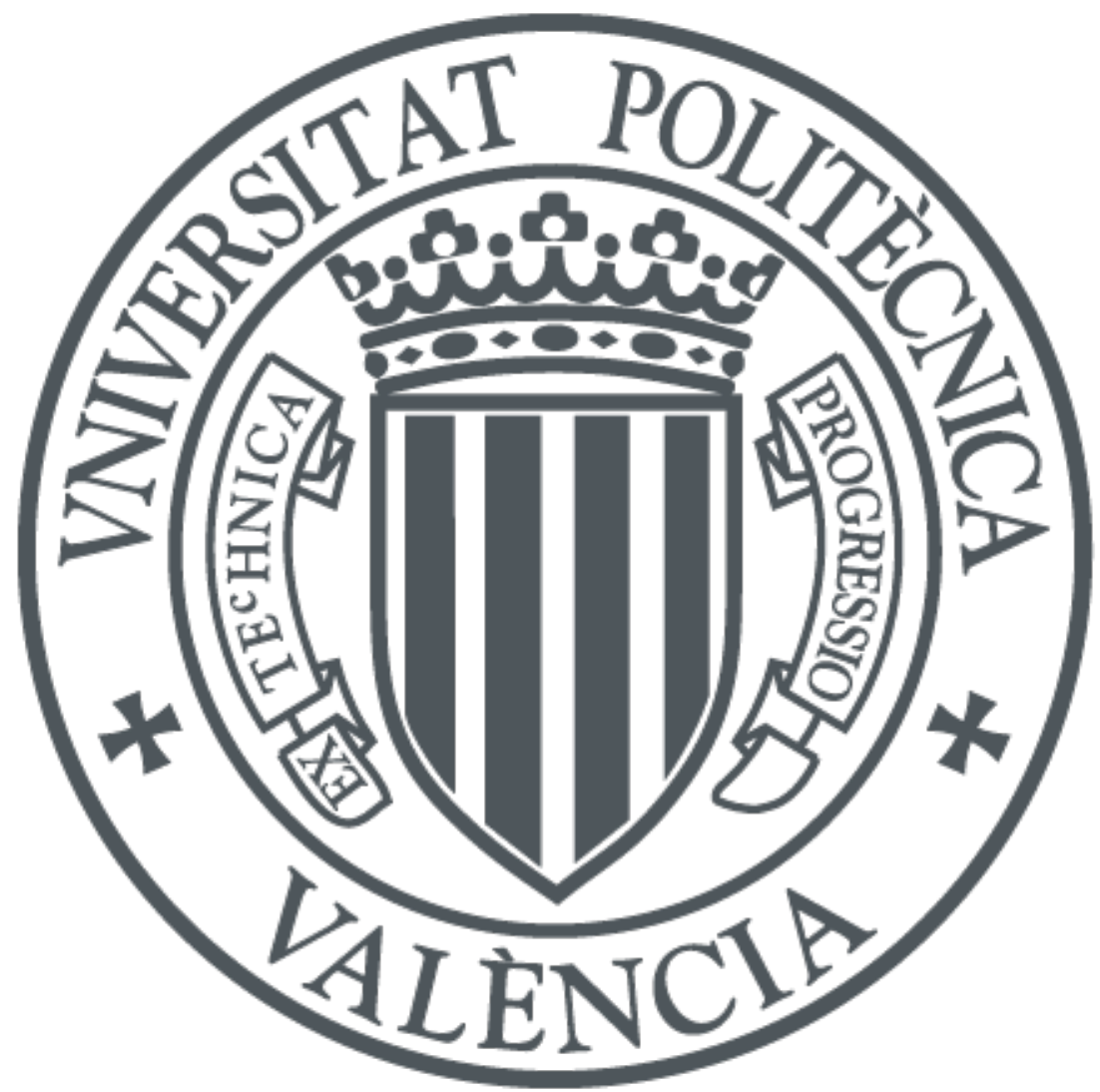

The final publication is available at

http://dx.doi.org/10.1145/2507924.2507935

Copyright ACM

Additional Information

CACM 2013. This is the author's version of the work. It is posted here for your personal use. Not for redistribution. The definitive Version of Record was published in MSWiM '13 Proceedings of the 16th ACM international conference on Modeling, analysis \& simulation of wireless and mobile systems; http://dx.doi.org/10.1145/2507924.2507935. 


\section{On the Selection of Optimal Broadcast Schemes in VANETs}

\author{
Julio A. Sanguesa, Manuel Fogue, \\ Piedad Garrido, Francisco J. Martinez \\ University of Zaragoza, Spain \\ jsanguesa, mfogue, piedad, \\ f.martinez\}@unizar.es
}

\author{
Juan-Carlos Cano, Carlos T. Calafate, \\ Pietro Manzoni \\ Universitat Politècnica de València, Spain \\ jucano, calafate, \\ pmanzoni\}@disca.upv.es
}

\begin{abstract}
In Vehicular ad hoc Networks (VANETs), efficient dissemination of messages is a key factor to speed up the development of useful services and applications. In this paper, we propose a novel algorithm that automatically chooses the best dissemination scheme trying to fit the warning message delivery policy to the current characteristics of each specific vehicular scenario. Our mechanism uses as input parameters the vehicular density and the topological characteristics of the environment where the vehicles are located, in order to decide which dissemination scheme to use. Simulation results demonstrate the feasibility of our approach, which is able to support more efficient warning message dissemination in vehicular environments.
\end{abstract}

\section{Categories and Subject Descriptors}

C.2.1 [Computer-Communication Networks]: Network Architecture and Design-Wireless communication

\section{General Terms}

Experimentation, Performance

\section{Keywords}

Vehicular ad hoc networks, warning message dissemination, adaptive systems, vehicular density estimation, VANETs

\section{INTRODUCTION}

Vehicular ad hoc Networks (VANETs) are wireless communication networks supporting cooperative driving among vehicles on the road. Vehicles act as communication nodes and relays, forming dynamic vehicular networks together with other nearby vehicles [1].

The specific characteristics of VANETs favor the development of attractive and challenging services and applications, including road safety, traffic flow management, road status monitoring, environmental protection, and mobile infotainment $[8,16]$. In this work we focus on traffic safety and efficient warning message dissemination, where the main goal is to reduce the latency and to increase the accuracy of the information received by nearby vehicles when a dangerous situation occurs.

In a VANET, any vehicle detecting an abnormal situation (i.e. accident, slippery road, etc.) rapidly starts notifying the anomaly to nearby vehicles to spread the alert information in a short period of time. Hence, broadcasting warning messages can be useful to alert nearby vehicles. However, this dissemination is strongly affected by: (i) the signal attenuation due to the distance between the sender and receiver (especially in low vehicular density areas), (ii) the effect of obstacles in signal transmission (very usual in urban areas, e.g., due to buildings), and (iii) a reduced message delivery effectiveness due to serious redundancy, contention, and massive packet collisions provoked by simultaneous forwarding, usually known as broadcast storm (prone to occur in highly congested areas) [14].

We consider that adapting the dissemination policy to the specific environment, accounting for the current vehicular density as well as for the scenario where the vehicles are located, can be beneficial in order to reduce broadcast storm related problems, and also to increase the efficiency of the warning message dissemination process.

In this paper, we propose an adaptive algorithm that automatically chooses the best dissemination scheme to adapt the warning message delivery policy to each specific scenario. Our mechanism uses as input parameters the vehicular density and the topological characteristics of the environment where the vehicles are located, using them to decide which dissemination scheme to use. The main goal is to maximize the message delivery effectiveness while generating a reduced number of messages and, thus, avoiding or mitigating broadcast storms. In addition, we also propose the Nearest Junction Located (NJL), our novel warning message dissemination scheme specially designed for being used in highly congested urban areas.

The paper is organized as follows: in Section 2 we review previous works closely related to our proposal, highlighting the main similarities and differences. In Section 3 we introduce our novel NJL scheme, and the optimal broadcast selection algorithm. Section 4 shows the simulation environment used to validate our proposal. Section 5 presents and discusses the obtained results. Finally, Section 6 concludes this paper. 


\section{RELATED WORK}

In the networking literature we can find several works that present adaptive mechanisms specially designed to enhance message dissemination in vehicular communications. In this section we present some of the most representative works.

Xue-wen et al. [15] proposed the Transmission Range Adaptive Broadcast (TRAB), a broadcast algorithm for VANETs. Considering the transmission ranges of vehicles together with the inter-vehicle distances, TRAB calculates the waiting time to select the relay vehicles in accordance with the additional coverage area of adjacent vehicles to ensure that fewer relay vehicles will be used to forward the emergency packets. In addition, TRAB guarantees the dissemination reliability by adopting two types of answering mechanisms called implicit ACK and explicit ACK. However, this scheme is designed to obtain efficient propagation of warning messages in highway scenarios alone, making it unsuitable for scenarios with complex topologies where we would want to disseminate warning messages in all directions surrounding the critical area.

Slavik et al. [12] proposed the Rate-Adaptive Broadcast (RAB) protocol for information dissemination in VANETs. $\mathrm{RAB}$ adapts to the network conditions, although it does not require any knowledge of network topology. By assuming a VANET dissemination application with fixed periodic updates, RAB is able to use a decision threshold control algorithm based on the rate of both messages. It tracks both the rate of received new messages and duplicate messages received. If the new message rate dips below its long-run average, the decision threshold is adjusted to improve message propagation. Otherwise, RAB adjusts the decision threshold to keep the duplicate message rate within an efficient range. Thus, RAB jointly optimizes the broadcast message delivery rate and the bandwidth consumption. Unlike the TRAB scheme, the use of RAB is not restricted to highways; nevertheless, the roadmap layout is not used to select the vehicles to forward the messages. Instead, the scenario is treated like a free space environment where vehicles only try to send messages as far away as possible, without accounting for the different blind areas that buildings may produce during the dissemination process.

Sommer et al. [13] proposed the Adaptive Traffic Beacon (ATB), a fully distributed message dissemination protocol which uses adaptive beaconing based on two key metrics: message utility and channel quality. Authors show that adaptive beaconing leads to a much broader dissemination of messages (in terms of penetration rate) than flooding-based approaches, although at a slower rate. The main objective of ATB is to exchange information in knowledge bases by sending beacons as frequently as possible, while maintaining a congestion-free wireless channel. However authors only tested their proposal in a roadmap portion of Ingolstadt, Germany.

Schwartz et al. [11] proposed a data dissemination protocol for VANETs that distributes data utility fairly over vehicles while adaptively controlling the network load. The protocol relies only on local knowledge to achieve fairness with concepts of Nash Bargaining from game theory. Simulation results show that their algorithm presents a higher fairness index, and it maintains a high level of bandwidth utilization efficiency compared to other approaches. In addition, the rate of transmissions is adaptively controlled as new information about the environment is collected. How- ever, the vehicular density of the scenarios where their proposal was tested was very low (i.e., only 20 vehicles $/ \mathrm{km}^{2}$ ). Additionally, it is not clearly explained if their simulations accounted for the effect of obstacles in wireless signal propagation, and the benefits of their proposal in terms of vehicles informed.

As shown, existing adaptive dissemination techniques for VANETs usually consider features related to vehicles in the scenario, such as their density, speed, and location, to adapt the performance of the dissemination process. However, most of the works in the literature are designed for highway scenarios where messages are only propagated in one direction, or focused on end-to-end routing. Additionally, most of them do not account for the effect of buildings and other obstacles during the dissemination of messages, which may lead to wrong conclusions. Hence, these approaches are not useful when attempting to warn the highest possible number of vehicles about dangerous situations in realistic vehicular environments. We consider that new adaptive proposals for warning message dissemination in urban environments are needed, offering efficient broadcasting techniques around the affected area, taking into account both the number of nearby vehicles, as well as the topology of the scenario.

\section{SELECTING THE OPTIMAL BROADCAST SCHEME IN VANETS}

Over the years, several schemes have been proposed to address the broadcast storm problem in vehicular networks. Some of the most representative ones are presented below.

\subsection{Broadcast Schemes}

- The Counter-based scheme [14]. Initially proposed for Mobile Ad Hoc Networks (MANETs), this scheme aims at mitigating broadcast storms by using a threshold $C$ and a counter $c$ to keep track of the number of times a broadcast message is received. Whenever $c \geq C$, rebroadcast is inhibited.

- The Distance-based scheme [14]. This scheme accounts for the relative distance $d$ between vehicles to decide whether to rebroadcast or not. When the distance $d$ between two vehicles is short, the additional coverage $(\mathrm{AC})$ area of the new rebroadcast is lower, and so rebroadcasting the warning message is not recommended. Forwarding is only beneficial when the additional coverage is nearly maximum.

- The enhanced Street Broadcast Reduction (eSBR) [6]. This scheme is specially designed to be used in VANETs, taking advantage of the information provided by maps and built-in positioning systems, such as the GPS. Vehicles are only allowed to rebroadcast messages if they are located far from their source $\left(>d_{\min }\right)$, or if the vehicles are located in different streets, giving access to new areas of the scenario. The eMDR scheme uses information about the roadmap to avoid blind areas due the presence of urban structures blocking the radio signal.

- The enhanced Message Dissemination for Roadmaps (eMDR) [4]. As an improvement to the eSBR scheme, eMDR increases the efficiency of the system by avoiding multiple forwardings of the same message if nearby 


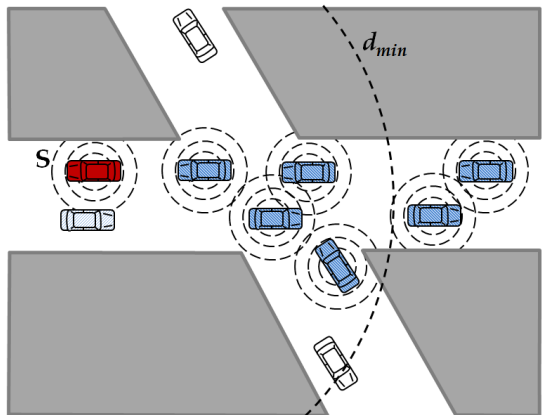

(a)

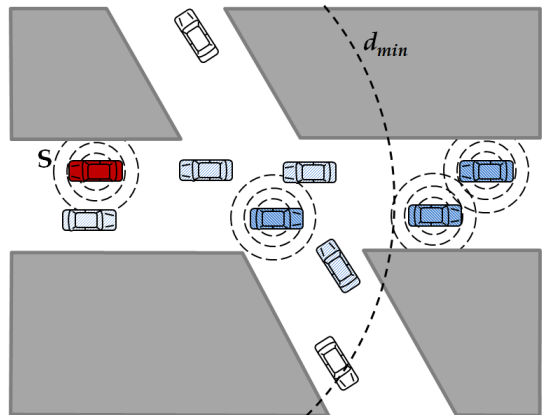

(b)

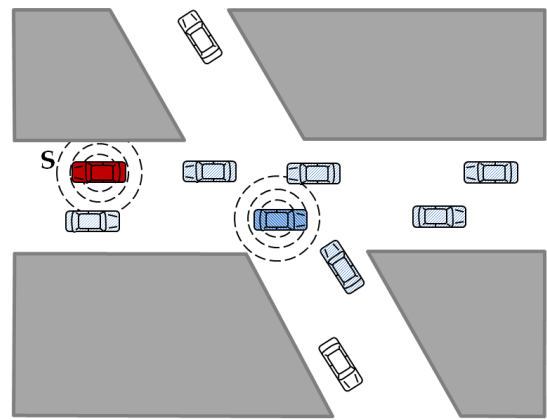

(c)

Figure 1: Comparison of different dissemination schemes for VANETs: (a) eSBR, (b) eMDR, and (c) NJL.

vehicles are located in different streets. Specifically, vehicles use the information about the junctions of the roadmap, and only the vehicle closest to the geographic center of the junction, according to the geopositioning system, is allowed to forward the messages received. This strategy aims at reducing the number of broadcasted messages while maintaining a high percentage of vehicles informed.

\subsection{Nearest Junction Located: our Novel Broad- cast Scheme}

The eMDR and eSBR schemes proved to be specially effective in sparse urban environments. However, the number of messages produced may become excessive in scenarios with a high vehicle density. To cope with this deficiency, in this paper we proposed a novel dissemination scheme called Nearest Junction Located (NJL) that is completely based on the topology of the roadmap, allowing vehicles to rebroadcast a message only if they are the nearest vehicle to the geographical coordinates of any junction obtained from the integrated maps. This scheme follows a procedure similar to the eMDR algorithm, although ignoring the distance between sender and receiver; thus, it only focus on the location of the receiving vehicle. Although the performance of this algorithm is not optimal in sparse environments, it performs quite well in high-density scenarios where the dominant factor to improve the dissemination process is the position of the vehicles, achieving results similar to those obtained by the eMDR and eSBR schemes, while requiring only a fraction of the messages.

Figure 1 shows the differences between the eSBR, eMDR and NJL schemes in a specific VANET scenario, where vehicle $S$ broadcasts a warning message. The line labeled as $d_{\text {min }}$ represents the minimum rebroadcast distance used by eSBR and eMDR. Darker vehicles will be allowed to forward the messages received from $S$, and it is noticeable how the eSBR is the less restrictive scheme, whereas the NJL is the most restrictive one, and thus more suitable for scenarios with a high vehicle density.

\subsection{Optimal Broadcast Selection Algorithm}

To select the optimal broadcast scheme for a specific scenario, we must account for different performance metrics, which will allow us to determine which scheme is the most efficient. These metrics should be quantitative to be easily adapted to different scenarios and environments, i.e., highways and urban maps.

During a warning message dissemination process, the most important objective to accomplish consists on informing the highest possible number of vehicles in the shortest time. Hence, a critical metric to be used is the percentage of informed vehicles at different time instants $\left(I n f_{T}\right)$. We propose to measure the percentage of vehicles receiving warning messages after 10, 30, and 120 seconds since the time when the dangerous situation started being notified, providing information about both the speed and completeness of the dissemination process. The first 10 seconds provide a good reference of the dissemination speed, the second period (30 seconds) offers a balance between dissemination speed and the completeness, and the state of the scenario after 120 seconds shows the stationary value when no evolution is observed.

These three values were combined using a weighted average, thereby obtaining a single value representing the efficiency of the dissemination process $\left(P_{i n f}\right)$. In our results, the weights applied to the values collected during the different time intervals are 0.5 (10 seconds), 0.3 (30 seconds), and 0.2 (120 seconds), respectively, since the stationary values of the different broadcast schemes do not tend to vary significantly, and the most noticeable differences occur during the first seconds of the process.

Another important metric for the dissemination schemes is the number of messages produced $\left(M_{\text {recv }}\right)$. If the wireless channel is saturated with packets, the high contention and the occurrence of collisions will reduce the performance of the process, producing broadcast storms. Hence, the number of messages must remain as low as possible without compromising the efficiency of the dissemination.

Our Optimal Broadcast Selection Algorithm makes use of these two metrics ( $P_{i n f}$ and $M_{\text {recv }}$ ) to select the scheme to be used on each particular situation. It works following a three step process, as shown in Algorithm 1:

- Step 1: For each considered broadcast scheme, the first metric $\left(P_{\text {inf }}\right)$ is computed, and the scheme with the highest percentage of informed vehicles in the shortest time is selected. Due to the importance of this metric, only the dissemination schemes with a deviation lower than $10 \%$ with respect to the best one are considered for the second step of the algorithm, and they are stored in set $\mathbb{C}$. 


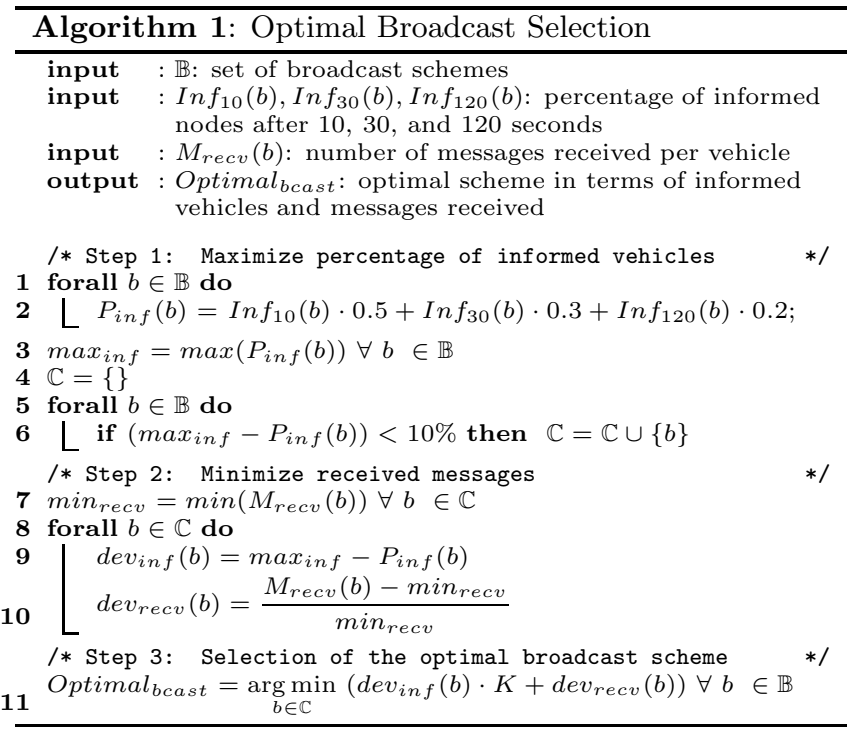

- Step 2: Considering only the broadcast schemes in $\mathbb{C}$, the scheme producing the lowest number of messages per vehicle $\left(M_{\text {recv }}\right)$ is obtained, in order to reduce the probability of broadcast storms, and the percentage variation with respect to this value is computed for each scheme.

- Step 3: The optimal scheme will be selected as the one minimizing the deviation with respect to both the maximal $P_{\text {inf }}$ and the minimal $M_{r e c v}$. Depending on the vehicle density, it may become more important to minimize the number of messages for high densities, and in that case our algorithm varies the degree of importance of the two metrics by using the $K$ value, calculated as follows:

$$
K=\frac{100}{\text { density of vehicles }}
$$

In particular, we used the value of reference 100 to compute $K$, since our experiments showed that the differences in terms of informed vehicles decrease noticeably for densities above 100 vehicles $/ \mathrm{km}^{2}$ (see Figure 2), and, hence, a higher weight is assigned to the number of messages received when this density is exceeded.

\section{SIMULATION ENVIRONMENT}

Our optimal broadcast selection algorithm was tested using the ns-2 simulator [2], modified to consider the IEEE $802.11 \mathrm{p}$ standard ${ }^{1}$. In terms of the physical layer, the data rate used for packet broadcasting is $6 \mathrm{Mbit} / \mathrm{s}$, as this is the maximum rate for broadcasting in 802.11 p. The MAC layer was also extended to include four different channel access priorities. Therefore, application messages are categorized into four different Access Categories (ACs), where AC0 has the lowest and AC3 the highest priority.

\footnotetext{
${ }^{1}$ All these improvements and modifications are available in http://www.grc.upv.es/software/
}

Table 1: Parameter settings in the simulations.

\begin{tabular}{|l|c|}
\hline Parameter & Value \\
\hline \hline roadmaps & Rome, Valencia, Sydney, \\
& Amsterdam, Los Angeles, \\
number of vehicles per $\mathrm{km}^{2}$ & San Francisco, Madrid \\
number of collided vehicles & 3 \\
roadmap size & $2000 \mathrm{~m} \times 2000 \mathrm{~m}$ \\
warning message size & $256 \mathrm{~B}$ \\
beacon message size & $512 \mathrm{~B}$ \\
warning messages priority & $A C 3$ \\
beacon priority & $A C 1$ \\
interval between messages & 1 second \\
MAC/PHY & $802.11 \mathrm{p}$ \\
radio propagation model & $R A V[7]$ \\
mobility model & $K r a u s s[5]$ \\
channel bandwidth & $6 M b p s$ \\
max. transmission range & $400 \mathrm{~m}$ \\
$d_{m i n}$ (used in distance-based, & $200 \mathrm{~m}$ \\
eSBR, and eMDR schemes) & \\
\hline
\end{tabular}

The simulator was also modified to make use of our Real Attenuation and Visibility (RAV) scheme [7], which proved to increase the level of realism in VANET simulations using real urban roadmaps in the presence of obstacles. As for vehicular mobility, it has been obtained with CityMob for Roadmaps (C4R) [3], a mobility generator able to import maps directly from OpenStreetMap [9], and make them available for being used by the ns- 2 simulator.

With regard to data traffic, vehicles operate in two modes: (a) warning mode, and (b) normal mode. Warning mode vehicles inform other vehicles about their status by sending warning messages periodically with the highest priority at the MAC layer; each vehicle is only allowed to propagate them once for each sequence number. Normal mode vehicles enable the diffusion of these warning packets and, periodically, they also send beacons with information such as their positions, speed, etc. These periodic messages have lower priority than warning messages, and so they are not propagated by other vehicles. All the results represent an average of over 50 repetitions with different random scenarios, obtaining for all of them a degree of confidence of $90 \%$; each simulation run lasted for 120 seconds. Table 1 shows the parameters used for the simulations.

The roadmaps used in the simulations were selected in order to have different profile scenarios (i.e., with different topology characteristics). Table 2 shows the main features of the cities simulated. Note that we added a column labeled as SJ Ratio, which represents the result of dividing the number of streets between the number of junctions. As shown, the first 4 cities (Rome, Valencia, Sydney, and Amsterdam) present an SJ ratio greater than 1, which indicates that they have a complex topology, while the rest of the cities (Los Angeles, San Francisco, and Madrid) present a lower SJ value, which indicates that they have a simple topology.

We are interested in the following performance metrics: (i) percentage of informed vehicles, and (ii) number of messages received per vehicle. The percentage of informed vehicles is the percentage of vehicles that do receive the warning messages sent by warning mode vehicles. The number of messages received per vehicle (including beacons and warning messages) gives an estimation of channel contention and the overhead of the selected approach. 
Table 2: Map features.

\begin{tabular}{|c|c|c|c|}
\hline Map & Streets & Junctions & SJ Ratio \\
\hline \hline Rome & 1655 & 1193 & 1.387 \\
\hline Valencia & 2829 & 2233 & 1.267 \\
\hline Sydney & 872 & 814 & 1.071 \\
\hline Amsterdam & 1494 & 1449 & 1.031 \\
\hline Los Angeles & 287 & 306 & 0.938 \\
\hline San Francisco & 725 & 818 & 0.886 \\
\hline Madrid & 628 & 715 & 0.878 \\
\hline
\end{tabular}

Table 3: Simulation Results in San Francisco after 120 seconds.

\begin{tabular}{|c|c|c|c|c|}
\hline & \multicolumn{2}{|c|}{25 veh. $/ \mathrm{km}^{2}$} & \multicolumn{2}{|c|}{250 veh. $/ \mathrm{km}^{2}$} \\
\hline & $\%$ informed & mess./veh. & $\%$ informed & mess./veh. \\
\hline$\overline{\text { eSBR }}$ & $\overline{89.9 \%}$ & $\overline{345}$ & $\overline{999.9 \%}$ & $4 \overline{4661}$ \\
\hline eMDR & $89.5 \%$ & 301 & $99.9 \%$ & 4275 \\
\hline$\overline{\text { NJL }}$ & $83.9 \%$ & 174 & $99.9 \%$ & 2184 \\
\hline
\end{tabular}

\section{SIMULATION RESULTS}

In this work we performed a total of 10,500 experiments, since we made 50 repetitions while also varying the city roadmaps, the density of vehicles, and the broadcast scheme used. Due to space restrictions, it is not possible to present the results of all of the cities simulated, so in some cases we only included the results obtained for San Francisco and Valencia since, according to our previous work [10], the simulation results obtained in these roadmaps are closer to the average ones.

In this section, we present: (i) the performance results of our proposed NJL broadcast scheme, (ii) a comparison between the studied broadcast schemes in terms of informed vehicles, (iii) a comparison in terms of messages received per vehicle, and finally, (iv) the performance of our Optimal Broadcast Selection algorithm.

\subsection{Performance of the Nearest Junction Lo- cated Scheme}

Tables 3 and 4 compare the simulation results after 120 seconds in two different maps (San Francisco and Valencia). The values of the percentage of informed vehicles and the number of messages received per vehicle are shown. As can be seen, the NJL scheme allows informing about 3-6\% less vehicles under low densities $\left(25\right.$ veh. $\left./ \mathrm{km}^{2}\right)$ in both maps, but the percentage of informed vehicles when the vehicle density is high is the same. However, the number of messages received per vehicle is reduced by half in all the scenarios tested using the NJL scheme. This makes the NJL scheme specially suitable for scenarios with a high density of vehicles where broadcast storms are prone to occur. It would be beneficial to use less restrictive schemes under low vehicle density conditions, such as the eSBR and the eMDR, to reach as many vehicles as possible since the number of messages produced is too low to produce broadcast storms.

\subsection{Comparison in Terms of Percentage of In- formed Vehicles}

As mentioned earlier, the most important metric to be considered when designing a warning message dissemination scheme for VANETs is the percentage of notified vehicles. We performed several experiments using roadmaps with different features and varying the density of vehicles. Figure 2
Table 4: Simulation Results in Valencia after 120 seconds.

\begin{tabular}{|c|c|c|c|c|}
\hline & \multicolumn{2}{|c|}{25 veh. $/ \mathrm{km}^{2}$} & \multicolumn{2}{|c|}{250 veh. $/ \mathrm{km}^{2}$} \\
\hline & $\%$ informed & mess./veh. & $\%$ informed & mess./veh. \\
\hline$\overline{\text { eSBR }}$ & $\overline{40.6 \%}$ & $\overline{55}$ & $\overline{999.7 \%}$ & $\overline{3360}$ \\
\hline eMDR & $38.8 \%$ & 48 & $99.7 \%$ & 2451 \\
\hline NJL & $37.4 \%$ & 41 & $99.7 \%$ & 1521 \\
\hline
\end{tabular}

presents the evolution of the dissemination process in terms of notified vehicles for the maps of San Francisco and Valencia under four different vehicle densities: 25, 100, 150, and 250 vehicles $/ \mathrm{km}^{2}$.

It is noticeable how the topology of the area and the number of vehicles are determinant factors affecting the performance of the broadcast scheme. The dissemination process develops faster in every situation when the vehicle density increases. For sparse networks, the counter-based scheme provides the best results in terms of informed vehicles, whereas for densities above 150 vehicles $/ \mathrm{km}^{2}$, the process presents a very similar behavior for all the selected schemes. The exception is the distance-based scheme in the map of Valencia, which proved to be very inefficient due to the high amount of obstacles interfering with the radio signal.

In addition, we corroborated that simple and regular city profiles like San Francisco allow an easier propagation of the radio signal, increasing the number of informed vehicles at a given time. The most restrictive schemes, such as the NJL, require a very high density of vehicles to achieve an efficiency similar to other dissemination schemes.

These results indicate that basing the selection only on the percentage of vehicles informed could lead to wrong decisions that could affect the efficiency of the system, justifying the need for additional metrics to perform the broadcast scheme selection.

\subsection{Comparison in Terms of Messages Received per Vehicle}

The number of messages produced by a given dissemination scheme may become very important in VANETs due to the high number messages sent and received by the vehicles involved. This could increase channel contention and the frequency of collisions. Therefore, a reduction of the number of messages sent under this situation would improve the warning message dissemination process, allowing other applications sharing the channel to operate adequately. To this end, it is necessary to evaluate the different dissemination schemes, taking into account the number of messages received by each vehicle in order to select the optimal scheme for each specific situation.

Figure 3 shows the number of messages received per vehicle in two of the maps under study. As shown, the selected dissemination scheme presents a determinant influence over the amount of messages produced; some of them produce only a fraction of the messages required by other schemes. In general, the counter-based scheme produces the highest number of messages, whereas the distance-based is the most restrictive one. As we might suppose, the NJL scheme produces the smallest amount of messages of all the schemes which used the information topology of the map to select the forwarding nodes. 


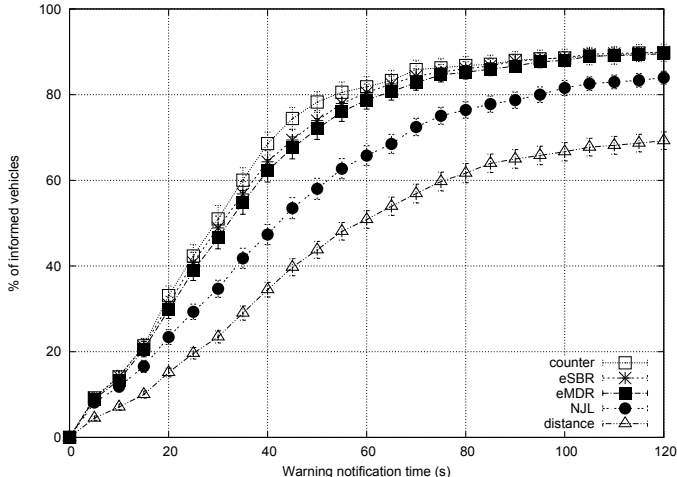

(a)

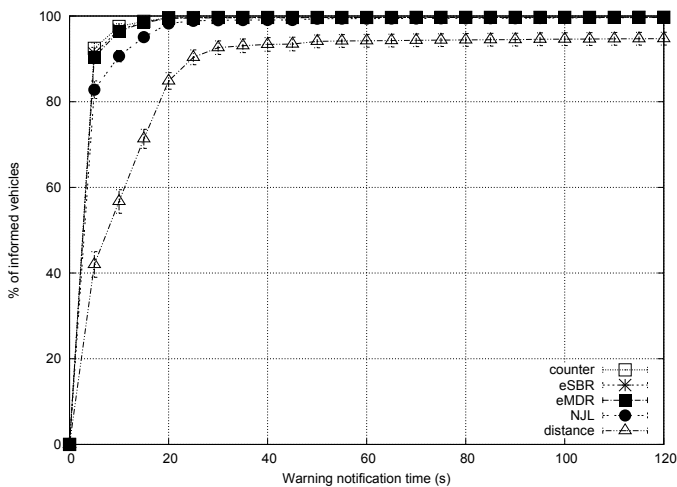

(b)

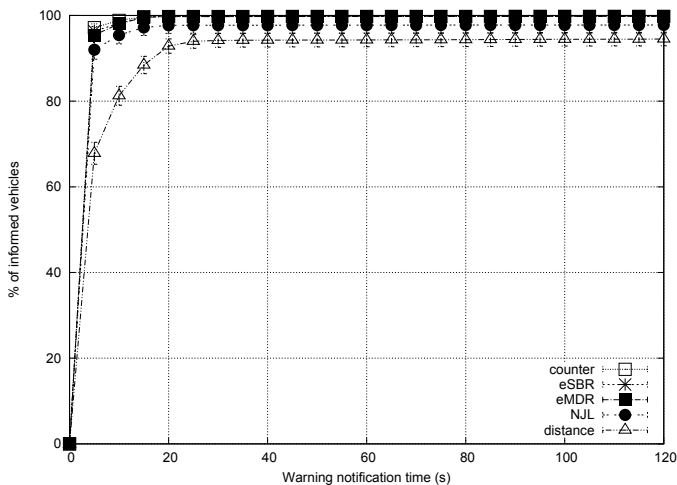

(c)

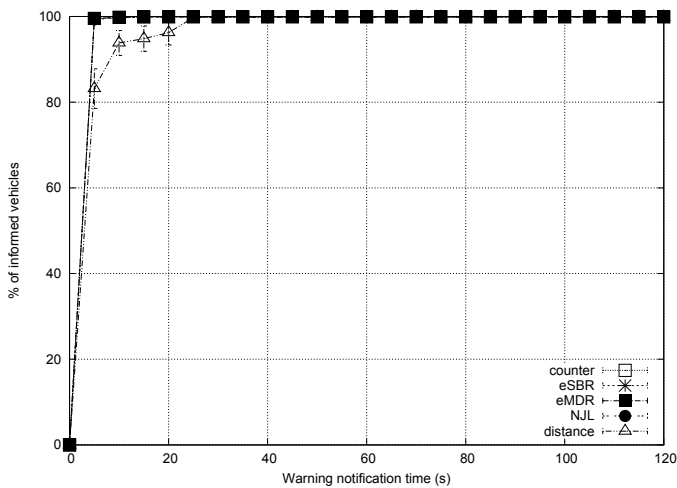

(d)

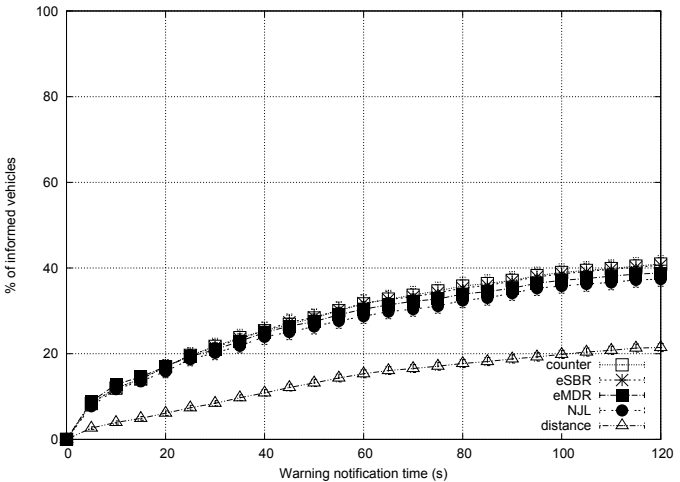

(e)

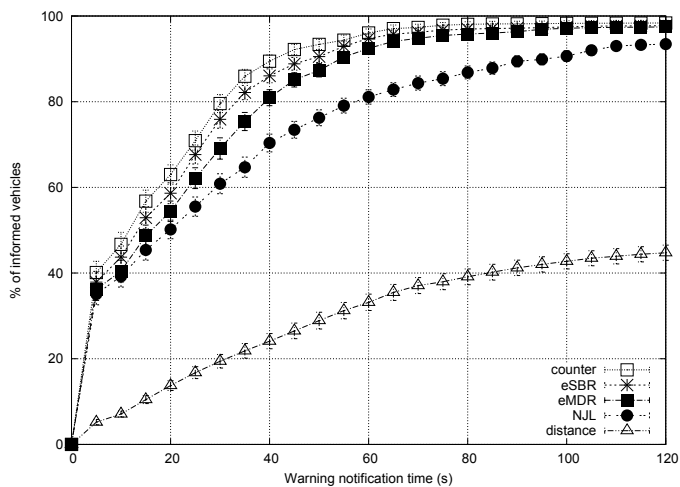

(f)

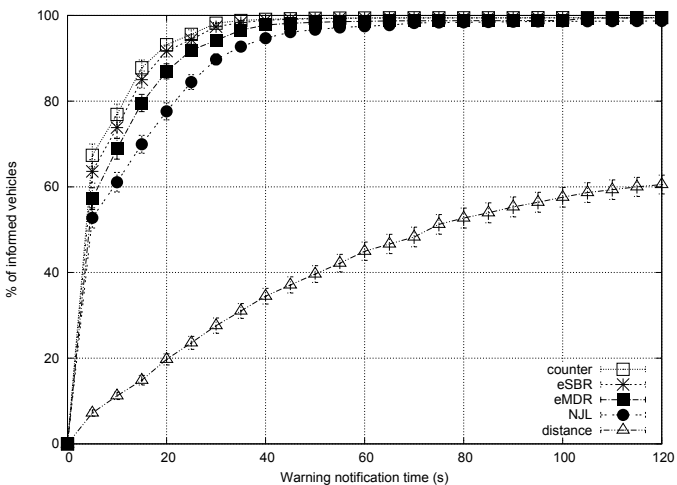

(g)

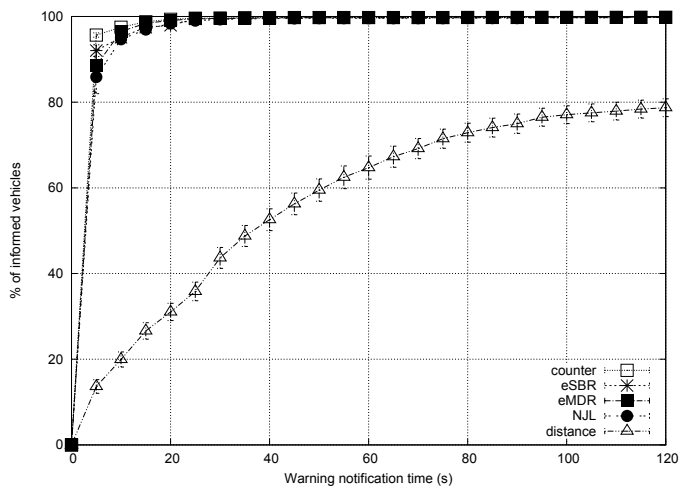

(h)

Figure 2: Percentage of informed vehicles in San Francisco for: (a) 25, (b) 100, (c) 150, and (d) 250 vehicles $/ \mathrm{km}^{2}$, as well as in Valencia for: (e) 25 , (f) 100, (g) 150, and (h) 250 vehicles $/ \mathrm{km}^{2}$. 


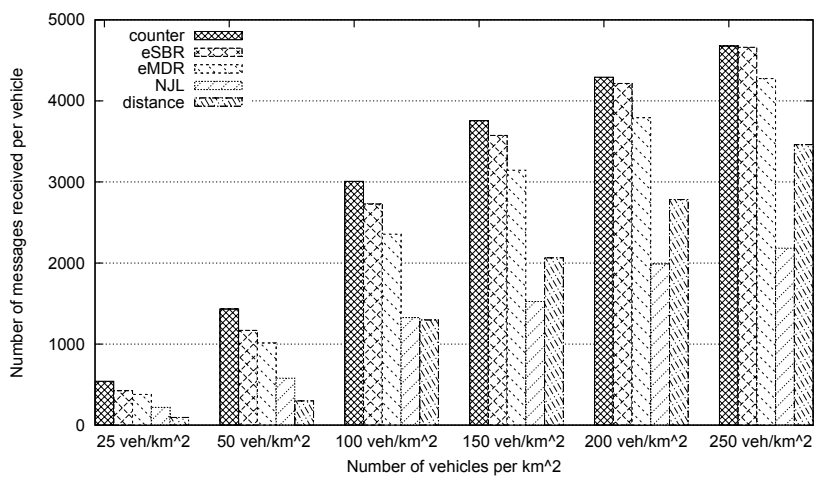

(a)

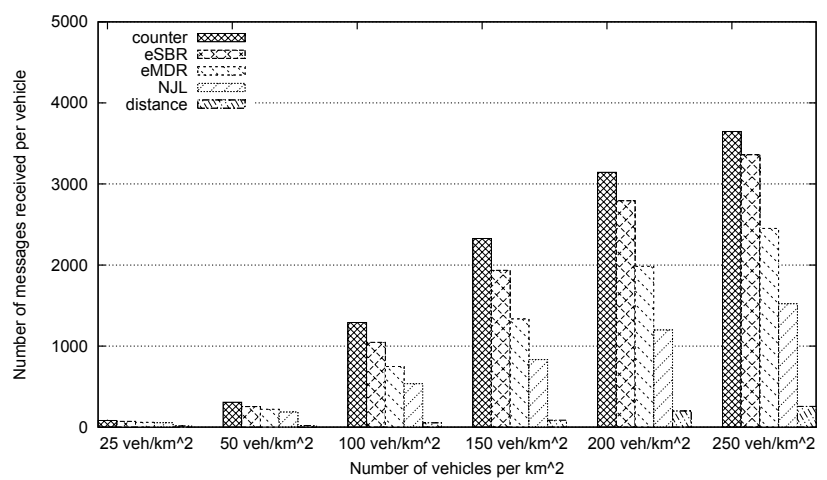

(b)

Figure 3: Number of messages received per vehicle when varying the broadcast scheme and the vehicular density in: (a) San Francisco and (b) Valencia.

Again, the features of the map are determinant for the performance of the system. Simple maps allow a faster dissemination at the cost of noticeably increasing the number of messages received per vehicle, thereby increasing the probability of broadcast storms. Thus, more restrictive schemes are recommended for this kind of roadmaps.

\subsection{Optimal Broadcast Scheme Selection}

Table 5 contains an example of the performance of our broadcast scheme selection algorithm presented in Section 3. Specifically, it shows the results obtained for Valencia when simulating 100 vehicles $/ \mathrm{km}^{2}$. All the values are obtained as the average of 50 repetitions for each configuration. It is noticeable how only three of the selected schemes are considered after the first step of the algorithm: i.e., the counter-based, the eSBR, and the eMDR broadcast schemes. Since the eMDR produces the lowest number of messages while maintaining a high percentage of informed vehicles in a small time period, our algorithm considers it as the optimal broadcast scheme for this specific situation.

Table 6 shows the selected broadcast scheme for each of the simulated scenarios according to our proposed Optimal Broadcast Selection Algorithm. Notice that the proposed NJL scheme is selected as the optimal one in most cases, especially under high vehicle densities or simple maps with a small SJ ratio, where the radio signal can reach long distances and broadcast storms are prone to occur. On the contrary, eMDR and eSBR schemes offer better results in scenarios where broadcast storms are not a problem, and the main objective is informing as many vehicles as soon as possible.

It is remarkable that almost all the schemes selected by our proposed algorithm rely on topology information to select the most appropriate forwarding vehicle, highlighting the importance of this factor in the warning dissemination process. In fact, broadcast schemes that only make use of the distance between the sender and the receiver, or which only focus on avoiding repeated messages, present a worse tradeoff between performance and the amount of messages required. We also observed an anomaly in the results obtained in Table 6 corresponding to the map of Madrid. The selected scheme when simulating 25 vehicles $/ \mathrm{km}^{2}$ is the counter- based one, while the overall trend indicates that the chosen one should be the eMDR scheme. This is due to the thresholds selected for Step 1 of the algorithm, where only those schemes with less than $10 \%$ variation with respect to the maximum value are considered. The eSBR and eMDR schemes achieve a value of $10.2 \%$ and $10.51 \%$ variation, respectively, which causes them to be ignored after the first step of the selection algorithm. This indicates that the use of fixed thresholds may lead to inaccurate decisions in some specific cases. We consider that a possible improvement of the broadcast selection algorithm could be using fuzzy logic to decide upon protocol adequacy, thereby avoiding those cases where values close to the threshold are completely ignored.

\section{CONCLUSIONS}

In this paper we proposed a quantitative algorithm that allows selecting the optimal broadcast scheme in a VANET scenario depending on two different metrics: (i) the percentage of informed vehicles, a particularly determinant factor in warning message dissemination, and (ii) the number of messages received by each vehicle, an important factor which indicates the channel contention and the possibility of broadcast storms during the dissemination of alert messages. In addition, we presented a new broadcast scheme called Nearest Junction Located (NJL), which was specially designed for scenarios presenting high vehicular densities or simple topologies, where broadcast storms are prone to occur. The NJL scheme is designed to reduce the number of messages received per vehicle without noticeably affecting the percentage of informed vehicles. We showed how our broadcast selection algorithm is able to select the optimal scheme in almost all the scenarios tested.

\section{ACKNOWLEDGMENTS}

This work was partially supported by the Ministerio de Ciencia e Innovación, Spain, under Grant TIN2011-27543C03-01, by the Fundación Universitaria Antonio Gargallo and the Obra Social de Ibercaja, under Grant 2013/B010, as well as the Government of Aragón and the European Social Fund (T91 Research Group). 
Table 5: Simulation results for 100 vehicles $/ \mathrm{km}^{2}$ in Valencia.

\begin{tabular}{|c|c|c|c|c|c|c|c|c|c|c|}
\hline Broadcast & $\operatorname{In} f_{10}$ & $\operatorname{Inf} f_{30}$ & $\operatorname{Inf_{120}}$ & $P_{\text {inf }}$ & $\operatorname{dev}_{i n f}$ & $\mathbb{C}($ Step 1) & $M_{\text {recv }}$ & $d_{\text {dev }}$ recv (Step 2) & $\operatorname{dev}_{T o t}$ & Optimal (Step 3) \\
\hline Counter & $=46.6 \%$ & $\overline{779.5 \%}$ & $\begin{array}{l}98.3 \% \\
\end{array}$ & "66.81 & $\overline{0 \%}$ & $\bar{v}$ & 1196 & $\overline{77.9 \%}$ & 75.55 & $\overline{\bar{x}}$ \\
\hline Distance & $7.10 \%$ & $19.4 \%$ & $44.7 \%$ & 18.31 & $72.59 \%$ & $\bar{x}$ & - & - & - & - \\
\hline eSBR & $43.7 \%$ & $75.8 \%$ & $97.7 \%$ & 64.13 & $4.01 \%$ & $\bar{v}$ & 940 & $39.87 \%$ & 43.89 & $\bar{x}$ \\
\hline eMDR & $40.4 \%$ & $69 \%$ & $97.4 \%$ & 60.38 & $9.62 \%$ & $\bar{v}$ & 672 & $0 \%$ & 9.62 & $\bar{v}$ \\
\hline NJL & $39.2 \%$ & $60.8 \%$ & $93.4 \%$ & 56.52 & $15.40 \%$ & $\bar{x}$ & - & - & - & - \\
\hline
\end{tabular}

Table 6: Broadcast Scheme Selected According to our Optimal Broadcast Selection Algorithm.

\begin{tabular}{|c|c|c|c|c|c|c|c|}
\hline \multirow{2}{*}{ City } & \multirow{2}{*}{ SJ Ratio } & \multicolumn{5}{|c|}{ Vehicle Density $\left(\mathbf{v e h} \cdot / \mathbf{k m}^{2}\right.$ ) } \\
\cline { 3 - 8 } & & 25 & 50 & 100 & 150 & 200 & 250 \\
\hline \hline Rome & 1.387 & eSBR & eSBR & eSBR & eSBR & NJL & NJL \\
\hline Valencia & 1.267 & eMDR & eMDR & eMDR & eMDR & NJL & NJL \\
\hline Sydney & 1.071 & eMDR & eMDR & eMDR & NJL & NJL & NJL \\
\hline Amsterdam & 1.031 & eMDR & eMDR & NJL & NJL & NJL & NJL \\
\hline Los Angeles & 0.938 & eMDR & eMDR & NJL & NJL & NJL & NJL \\
\hline San Francisco & 0.886 & eMDR & eMDR & NJL & NJL & NJL & NJL \\
\hline Madrid & 0.878 & Counter & eMDR & NJL & NJL & NJL & NJL \\
\hline
\end{tabular}

\section{REFERENCES}

[1] F. Ahammed, J. Taheri, A. Zomaya, and M. Ott. VLOCI2: improving 2D location coordinates using distance measurements in GPS-equipped VANETs. In Proceedings of the 14th ACM international conference on Modeling, analysis and simulation of wireless and mobile systems, MSWiM '11, pages 317-322, New York, NY, USA, 2011. ACM.

[2] K. Fall and K. Varadhan. ns notes and documents. The VINT Project. UC Berkeley, LBL, USC/ISI, and Xerox PARC, February 2000.

[3] M. Fogue, P. Garrido, F. J. Martinez, J.-C. Cano, C. T. Calafate, and P. Manzoni. A Realistic Simulation Framework for Vehicular Networks. In 5th International ICST Conference on Simulation Tools and Techniques (SIMUTools 2012), Desenzano, Italy, pages 37-46, March 2012.

[4] M. Fogue, P. Garrido, F. J. Martinez, J.-C. Cano, C. T. Calafate, and P. Manzoni. Evaluating the impact of a novel message dissemination scheme for vehicular networks using real maps. Transportation Research Part C: Emerging Technologies, 25:61-80, December 2012.

[5] S. Krauss, P. Wagner, and C. Gawron. Metastable states in a microscopic model of traffic flow. Physical Review E, 55(5):5597-5602, 1997.

[6] F. J. Martinez, M. Fogue, M. Coll, J.-C. Cano, C. Calafate, and P. Manzoni. Evaluating the impact of a novel warning message dissemination scheme for VANETs using real city maps. In M. Crovella, L. Feeney, D. Rubenstein, and S. Raghavan, editors, NETWORKING 2010, volume 6091 of Lecture Notes in Computer Science, pages 265-276. Springer Berlin / Heidelberg, 2010.

[7] F. J. Martinez, M. Fogue, C. K. Toh, J.-C. Cano, C. T. Calafate, and P. Manzoni. Computer simulations of VANETs using realistic city topologies. Wireless Personal Communications, 69(2):639-663, 2013.

[8] F. J. Martinez, C. K. Toh, J.-C. Cano, C. T. Calafate, and P. Manzoni. Determining the representative factors affecting warning message dissemination in
VANETs. Wireless Personal Communications, 67(2):295-314, November 2012.

[9] OpenStreetMap. Collaborative project to create a free editable map of the world, 2012. Available at http://www.openstreetmap.org.

[10] J. A. Sanguesa, M. Fogue, P. Garrido, F. J. Martinez, J.-C. Cano, C. T. Calafate, and P. Manzoni. An infrastructureless approach to estimate vehicular density in urban environments. Sensors, 13(2):2399-2418, 2013

[11] R. Schwartz, A. Ohazulike, C. Sommer, H. Scholten, F. Dressler, and P. Havinga. Fair and adaptive data dissemination for traffic information systems. In IEEE Vehicular Networking Conference, pages 1-8, 2012.

[12] M. Slavik, I. Mahgoub, and M. Alwakeel. Adapting statistical multi-hop wireless broadcast protocol decision thresholds using rate control. In 9th International Conference on High Capacity Optical Networks and Enabling Technologies (HONET), pages 32-36, 2012.

[13] C. Sommer, O. Tonguz, and F. Dressler. Traffic information systems: efficient message dissemination via adaptive beaconing. IEEE Communications Magazine, 49(5):173-179, 2011.

[14] Y.-C. Tseng, S.-Y. Ni, Y.-S. Chen, and J.-P. Sheu. The broadcast storm problem in a mobile ad hoc network. Wireless Networks, 8:153-167, 2002.

[15] W. Xue-wen, Y. Wei, S. Shi-ming, and W. Hui-bin. A transmission range adaptive broadcast algorithm for vehicular ad hoc networks. In Proceedings of the 2010 Second International Conference on Networks Security, Wireless Communications and Trusted Computing - Volume 01, NSWCTC '10, pages 28-32, Washington, DC, USA, 2010. IEEE Computer Society.

[16] X. Zhuo, J. Hao, D. Liu, and Y. Dai. Removal of misbehaving insiders in anonymous VANETs. In N. B. Abu-Ghazaleh, L. Bononi, B. Bensaou, and V. R. Syrotiuk, editors, Proceedings of the 12th ACM international conference on Modeling, analysis and simulation of wireless and mobile systems, MSWiM '09, pages 106-115, New York, NY, USA, 2009. ACM. 